
у пациентов с воспалительными заболеваниями кишечника в Новосибирской области: одноцентровое поперечное одномоментное исследование 245 больных

\author{
Краснер Я.А. • Кулыгина Ю.А. • Валуйских Е.Ю. • Осипенко М.Ф.'
}

Краснер Яков Аркадьевич - ассистент кафедры пропедевтики внутренних болезней лечебного факультета' $\triangle 630078$, г. Новосибирск, ул. Котовского, 5/1-13, Российская Федерация. Тел.: +7 (913) 9077766. E-mail: yakov.krasner@yandex.ru

Кулыгина Юлия Александровна ассистент кафедры пропедевтики внутренних болезней лечебного факультета

Валуйских Екатерина Юрьевна - канд. мед. наук, заведующая терапевтическим отделением²

Осипенко Марина Федоровна - д-р мед. наук, профессор, заведующая кафедрой пропедевтики внутренних болезней лечебного факультета'
Актуальность. В последнее время в развитых странах отмечается рост заболеваемости воспалительными заболеваниями кишечника (ВЗК) и неалкогольным стеатогепатозом. Вместе с тем существуют фундаментальные предпосылки к наличию взаимоотягощающего влияния этих заболеваний. В связи с этим изучение особенностей неалкогольного стеатогепатоза у больных ВЗК представляет существенный практический интерес. Цель - выявить особенности неалкогольного стеатогепатоза/стеатогепатита у пациентов с ВЗК. Материал и методы. В одномоментное исследование включены 245 пациентов в возрасте от 18 до 77 лет, состоящие в Регистре хронических воспалительных заболеваний кишечника ФГБОУ ВО НГМУ Минздрава России и НИИФФМ (г. Новосибирск, Российская Федерация). За год до включения в исследование пациентам выполняли ультразвуковое исследование (УЗИ) органов брюшной полости, по данным которого устанавливалось наличие стеатогепатоза. Все пациенты проходили клиническое обследование с определением антропометрических показателей, больным выполнялись лабораторные исследования: общий анализ крови, оценка уровней аланиновой и аспарагиновой трансаминаз, креатинина, триглицеридов, общего холестерина. Из исследования исключались пациенты с установленными диагнозами вирусного и аутоиммунного гепатита, алкогольной болезни печени, первичного склерозирующего холангита, нарушениями обмена железа и меди. У 45 больных в биоптатах из толстой кишки определялись вирусы Эпштейна - Барр, цитомегаловирус, вирус простого герпеса методом полимеразной цепной реакции. У 152 больных проводилась оценка синдрома избыточного бактериального роста (СИБР). Результаты. Частота стеатоза у пациентов с болезнью Крона (БК) составила
$34,2 \%$, а у пациентов с язвенным колитом (ЯК) - 30,4\%. Пациенты с ВЗК и стеатогепатозом чаще получали стероиды в анамнезе (63,6 против 53\%, $\mathrm{p}=0,0006)$, имели больший стаж основного заболевания (медиана 5,9 года; $\mathrm{Q}_{1} / \mathrm{Q}_{3}$ $2,7 / 12,9$ года против 4,5; 2,9/8,5 года; $p=0,0324)$ и больший индекс массы тела (медиана 24,1; $\mathrm{Q}_{1} / \mathrm{Q}_{3}-21,4 / 29,9$ против $21 ; 18,6 / 23,5 ; \mathrm{p}=0,0336$ ). У больных ЯК при наличии неалкогольного стеатоза/стеатогепатита был выше возраст (для возраста старше 40 лет отношение шансов (ОШ) составило 1,46; 95\% доверительный интервал (ДИ) 1,01-2,1). У пациентов с БК и стеатогепатозом уровень тромбоцитов был выше (медиана $287 \times 10^{9} /$ л; $\mathrm{Q}_{1} / \mathrm{Q}_{3}$ - $192 \times 10^{9} /$ л/420 $\times 10^{9} /$ л против медианы $250 \times 10^{9} /$ л; $180 \times 10^{9} /$ л/379 $\times 10^{9} / л$; $\mathrm{p}=0,0183)$. СИБР был фактором риска развития стеатогепатоза у больных В3К (ОШ 2,34; 95\% ДИ 1,4-4,8; $\quad$ =0,021). Заключение. Выявлены различия в факторах, ассоциированных со стеатогепатозом у пациентов с ЯК и БК. Продемонстрирована взаимосвязь между наличием СИБР и риском формирования стеатогепатоза у больных ВЗК.

Ключевые слова: воспалительные заболевания кишечника, болезнь Крона, язвенный колит, стеатогепатоз, микрофлора

Для цитирования: Краснер ЯА, Кулыгина ЮА, Валуйских ЕЮ, Осипенко МФ. Частота и особенности неалкогольного стеатогепатоза у пациентов с воспалительными заболеваниями кишечника в Новосибирской области: одноцентровое поперечное одномоментное исследование 245 больных. Альманах клинической медицины. 2018;46(5):464-73. doi: 10.18786/2072-0505-201846-5-464-473

Поступила 01.08.2018;

принята к публикации 02.10.2018 
$\mathrm{B}$ опросы, связанные со стеатозом печени, активно изучаются $[1,2]$. Как морфологический симптом стеатоз известен давно, долгое время он относился к разряду специфичных признаков алкогольного повреждения печени [3]. В последние два десятилетия стало ясно, что стеатоз/стеатогепатит формируется в результате различных патологических состояний: сахарного диабета, действия лекарственных препаратов, HCV-инфекции, особенно 3-го генотипа, аутоиммунных факторов, нарушений обмена железа и меди $[4,5]$.

Среди важнейших факторов, ассоциированных со стеатозом, выделяют метаболический синдром. Эта нозологическая форма носит название неалкогольной жировой болезни печени (НАЖБП) и также находится в центре внимания исследователей. При установлении диагноза НАЖБП одним из критериев выступает наличие стеатоза, доказанного визуализирующими методами (ультразвуковое исследование (УЗИ), компьютерная томография и др.), при исключении таких этиологических факторов, как алкоголь, и других вторичных причин, которые могут сопровождаться стеатозом [6].

Стеатоз печени изучается при различных заболеваниях внутренних органов с целью оценки взаимного влияния этих состояний $[7,8]$. В частности, вследствие роста заболеваемости и распространенности воспалительных заболеваний кишечника (ВЗК) проблема поражения печени, ранее сводившаяся к первичному склерозирующему холангиту и аутоиммунному гепатиту, приобрела особое звучание, в том числе в связи с появлением новых взглядов на патогенез болезней печени, а также гипотез о взаимном утяжелении течения ВЗК и стеатоза $[9,10]$. Более того, одним из ведущих факторов, ассоциированных с возникновением патологии печени, называют заболевания кишечника, сопровождающиеся нарушением интестинального барьера [11-13].

Учитывая высокий интерес к теме коморбидности патологии печени и кишечника, целью нашего исследования была оценка частоты стеатоза/стеатогепатита у больных ВЗК и определение факторов, ассоциированных со стеатозом/стеатогепатитом при ВЗК.

Следует отметить, что термин НАЖБП применяется при исключении определенных этиологических факторов, в число которых входят и лекарственные препараты. В то же время у пациентов с ВЗК достоверно исключить влияние принимаемых лекарств на формирование НАЖБП крайне сложно, поэтому во избежание терминологических неточностей в нашей работе мы будем использовать более широкое понятие «неалкогольный стеатогепатоз / стеатогепатит».

\section{Материал и методы}

В одномоментное поперечное исследование были включены 245 больных с диагнозом В3К в возрасте от 18 до 77 лет, средний возраст составил 38,5 $\left(\mathrm{Q}_{1} / \mathrm{Q}_{3}\right.$ - 29,75/52,25) года. Все больные состоят в регистре воспалительных заболеваний кишечника ФГБОУ ВО НГМУ Минздрава России и НИИФФМ («Регистр хронических воспалительных заболеваний кишечника», свидетельство о государственной регистрации базы данных № 2017620133, дата регистрации 03.02.2017). На момент начала исследования данный регистр включал 1260 человек, из них с болезнью Крона (БК) было 419, с язвенным колитом (ЯК) - 656, «неклассифицируемым колитом» - 185. Диагноз ВЗК устанавливался в соответствии с Клиническими рекомендациями Российской гастроэнтерологической ассоциации и Ассоциации колопроктологов России по диагностике и лечению язвенного колита (2017) и Клиническими рекомендациями Российской гастроэнтерологической ассоциации и Ассоциации колопроктологов России по диагностике и лечению болезни Крона $(2017)$ [14, 15]. В исследование включались пациенты, которым не ранее чем за год до даты включения выполнялось УЗИ органов брюшной полости.

Bce пациенты проходили детальное клиническое обследование, предполагавшее сбор жалоб и анамнеза, оценку особенностей клинического течения заболевания, всем пациентам рассчитывали индекс массы тела (ИМТ, кг/м²), измеряли окружность талии, проводили подробное физикальное обследование. Учитывали также данные ряда лабораторных и инструментальных исследований: общий анализ крови, оценка уровней аланиновой и аспарагиновой трансаминаз, гамма-глутамилтрансферазы, щелочной фосфатазы, билирубина, мочевины, креатинина. Оценивались показатели липидного обмена (уровни общего холестерина, триглицеридов, липопротеидов низкой и высокой плотности). Абдоминальное ожирение констатировали при окружности талии более 102 см у мужчин и 88 см у женщин. Все пациенты заполняли опросник AUDIT (Alcohol Use Disorders Identification Test) для выявления и исключения лиц, злоупотребляющих алкоголем. Наряду с этим проводилось обследование для исключения иных факторов, вызывающих поражение печени, HCV- и HBV-инфекций, тесты на аутоиммунный 
гепатит, обмен железа и меди. Из исследования исключались пациенты с установленными диагнозами вирусного и аутоиммунного гепатита, первичного склерозирующего холангита, алкогольной болезни печени, нарушениями обмена железа и меди.

Наличие стеатоза устанавливалось на основании данных УЗИ органов брюшной полости в соответствии с рекомендациями Российского общества по изучению печени (2015) при наличии следующих ультразвуковых диагностических критериев: диффузная гиперэхогенность паренхимы печени и неоднородность ее структуры, нечеткость и/или подчеркнутость сосудистого рисунка, более высокая эхогенность печени в сравнении с корковым веществом почек. При повышении уровня маркеров цитолиза устанавливался диагноз стеатогепатита [16].

у больных со стеатозом/стеатогепатитом проводилась оценка уровня фиброза по формулам APRI (Aspartate-aminotransferase-to-Platelet Ratio Index) и FIB4 (Fibrosis-4) [17]. У 45 больных в биоптатах из толстой кишки определялись вирусы Эпштейна - Барр, цитомегаловирус, вирус простого герпеса методом полимеразной цепной реакции.

У 152 больных проводилась оценка синдрома избыточного бактериального роста (СИБР). В исследование включались больные с установленным более 3 месяцев диагнозом ЯК или БК в возрасте от 18 до 75 лет. Критериями исключения были аллергия или непереносимость лактулозы; прием антибактериальных препаратов, пробиотиков, препаратов висмута в течение 4 недель до исследования и прокинетиков в течение 3 периодов полувыведения; длительный прием ингибиторов протонной помпы; эндоскопическое или рентгенологическое обследование пищеварительной системы в течение 4 недель до исследования; очищение кишечника в течение 4 недель до исследования; хронические заболевания, при которых может встречаться СИБР (панкреатит, цирроз печени, целиакия, сахарный диабет); наличие илеостомы, а также наличие тяжелых сопутствующих соматических заболеваний. Для диагностики СИБР использовался водородный дыхательный тест на аппарате «Gastro+» с лактулозой. При проведении теста, после измерения базального уровня водорода в выдыхаемом воздухе, всем пациентам давали выпить лактулозу в дозе 15 мл (10 г), растворенную в 200 мл воды. Измерение концентрации водорода в выдыхаемом воздухе проводилось натощак и через $15,30,60,90,120$ минут.
Положительным результатом считался уровень более 20 ррт при наличии двойного пика уровней водорода (первый в пределах 90 минут) или устойчивый рост более чем на 12 ppm по сравнению с исходным уровнем водорода (что свидетельствует о наличии СИБР).

Протокол исследования был одобрен локальным этическим комитетом ФГБОУ ВО НГМУ Минздрава России от 16.05.2017.

Статистические методы. С законом нормального распределения по критерию Шапиро Уилка согласованы 7\% от всех сравниваемых непрерывных показателей, поэтому для сравнения использовались непараметрические методы. Дескриптивные характеристики представлены в виде медианы; первый квартиль $\left(Q_{1}\right) /$ третий квартиль $\left(\mathrm{Q}_{3}\right)$ для непрерывных числовых данных. Для оценки корреляционных связей использовался коэффициент Спирмена. Для статистической проверки гипотез о равенстве числовых характеристик выборочных распределений в сравниваемых группах использовался непарный U-критерий Манна - Уитни, производился расчет смещения распределений с построением 95\% доверительного интервала (ДИ) для смещения. Отношение шансов (ОШ) с доверительными интервалами (95\% ДИ) рассчитывали по таблицам сопряженности. Для сравнения бинарных и категориальных показателей применялся точный двусторонний критерий Фишера. Для учета множественных сравнений применялась поправка Бонферрони. Проверка статистических гипотез проводилась при критическом уровне значимости $\mathrm{p}=0,05$, то есть различие считалось статистически значимым, если $\mathrm{p}<0,05$. Статистические расчеты проводились в программe Statistica 12.0, Rstudio (version 0.99.879 - (с) 20092016 RStudio, Inc., USA, 250 Northern Ave, Boston, MA 02210 844-448-121, info@rstudio.com) на языке R.

\section{Результаты и обсуждение}

Среди 245 больных, включенных в исследование, частота стеатоза (преимущественно по данным абдоминального УЗИ) составила $32,2 \%$. При этом статистически значимых различий частоты встречаемости стеатоза у пациентов с БК и ЯК выявлено не было $(\mathrm{p}=0,86)$. Так, частота стеатоза у пациентов с БК составила $34,2 \%$, а у пациентов с ЯК - 30,4\%.

В исследовании S. Bargiggia и соавт. [18] встречаемость стеатогепатоза, диагностированного по данным УЗИ органов брюшной полости, среди пациентов с БК составляет 39,5\%, с ЯК - 35,5\%. 
У $27,3 \%$ пациентов с БК и $24,5 \%$ с ЯК диагностирован стеатогепатоз легкой (небольшое увеличение эхогенности печени) и средней (отсутствие эхосигнала от стенки воротной вены и выраженные различия печени и почек по эхогенности) степеней. В последних опубликованных исследованиях частота НАЖБП у больных ВЗК равняется $32,8 \%$ (выраженного фиброза - 12,2\%) [9], $28 \%$ [19] и 33,6\% [20]. Таким образом, несмотря на некоторую вариативность данных, можно констатировать, что примерно у каждого третьего пациента с ВЗК имеются признаки неалкогольного стеатоза независимо от варианта течения - ЯК или БК.

По нашим данным, в целом в группе больных ВЗК при наличии неалкогольного стеатоза/стеатогепатита была больше длительность заболевания (время с момента возникновения первых симптомов болезни) (для длительности более 15 лет ОШ составило 1,36; 95\% ДИ 1,03-1,78), большее количество пациентов получали стероиды и в лечении применялась бо́льшая доза стероидов, был выше ИМТ (для ожирения ОШ равно 1,43; 95\% ДИ 1,12-1,81) (табл. 1). В группе больных стеатозом чаще встречались мужчины.

В недавно опубликованных исследованиях также предприняты попытки выявить ассоциированные с НАЖБП факторы. Так, P.C. Saroli и соавт. [9] в качестве независимых факторов НАЖБП вычленяют возраст (для старших возрастных групп ОШ равно 1,45; $95 \%$ ДИ 1,15-1,82), высокий ИМТ (ОШ 1,31; 95\% ДИ 1,2-1,42) и повышенный уровень триглицеридов (ОШ 1,45; 95\% ДИ 1,01-2,09). В работе М. Principi и соавт. [19] НАЖБП у больных ВЗК ассоциирована с метаболическим синдромом (ОШ 2,24; 95\% ДИ 1,7728,81), диабетом (ОШ 1,71; 95\% ДИ 1,43-12,25), нарушением толерантности к глюкозе (ОШ 1,36 ; $95 \%$ ДИ $1,13-1,68)$ и окружностью талии (ОШ 1,68 ; 95\% ДИ 1,15-14,52). A. Sartini и соавт. [20] не выявили явной взаимосвязи стеатоза у больных ВЗК с типичными признаками метаболического синдрома. Согласно результатам, полученным К. Glassner и соавт. [10], больные ВЗК со стеатозом старше, у них выше ИМТ, больше продолжительность течения болезни кишечника, выше риск диабета и ожирения по сравнению с пациентами с ВЗК без стеатоза. Однако пациенты с НАЖБП без ВЗК чаще имеют ожирение, гиперлипидемию и диабет.

Проведенный нами анализ факторов, ассоциированных со стеатозом/стеатогепатитом, у пациентов в зависимости от наличия ЯК или БК показал, что у больных ЯК с неалкогольным
Таблица 1. Сравнительная характеристика больных воспалительными заболеваниями кишечника в зависимости от наличия (1) или отсутствия (2) неалкогольного стеатоза / стеатогепатита $(n=93)$

\begin{tabular}{llll}
\hline Параметр & $1(\mathrm{n}=34)$ & $2(\mathrm{n}=59)$ & $p$ \\
\hline Доля мужчин, \% & 42 & 37 & 0,0311 \\
$\begin{array}{l}\text { Длительность заболевания, годы } \\
\left.\text { (медиана; } \mathrm{Q}_{1} / \mathrm{Q}_{3}\right)\end{array}$ & 5,$9 ; 2,7 / 12,9$ & 4,$5 ; 2,9 / 8,5$ & 0,0324 \\
$\begin{array}{l}\text { Максимальная доза стероидов, мг } \\
\left.\text { (медиана; } \mathrm{Q}_{1} / \mathrm{Q}_{3}\right)\end{array}$ & $20 ; 0 / 60$ & $10 ; 0 / 60$ & 0,0086 \\
$\begin{array}{l}\text { Доля пациентов, получавших } \\
\text { глюкокортикостероиды, \% }\end{array}$ & 63,6 & 53 & 0,0006 \\
\begin{tabular}{l} 
Индекс массы тела (медиана; $\left.\mathrm{Q}_{1} / \mathrm{Q}_{3}\right)$ \\
\hline
\end{tabular} & 24,$1 ; 21,4 / 29,9$ & $21 ; 18,6 / 23,5$ & 0,0336 \\
\hline
\end{tabular}

стеатозом/стеатогепатитом был выше возраст (для возраста более 40 лет ОШ составило 1,46; 95\% ДИ 1,01-2,1), ИМТ (при ожирении ОШ 1,37; 95\% ДИ 0,97-1,95); такие пациенты чаще получали глюкокортикостероиды и реже биологическую терапию. Не было выявлено статистически значимых различий по уровню гемоглобина, максимальному значению скорости оседания эритроцитов (СОЭ), уровню холестерина и целому ряду других параметров (табл. 2). В работе R. Jamali и соавт. [21] установлена взаимосвязь между расчетным показателем содержания липидов в печени и тяжестью ЯК. Между ультразвуковой стадией НАЖБП и тяжестью и протяженностью ЯК взаимосвязи не обнаружено.

Нами установлено, что больные БК при наличии неалкогольного стеатоза/стеатогепатита чаще получали биологическую терапию и реже азатиоприн (табл. 3). В нашем исследовании у пациентов с БК была выявлена статистически значимая ассоциация между уровнем тромбоцитов на момент обследования и наличием стеатогепатоза $\left(287 \times 10^{9} /\right.$ л у пациентов со стеатогепатозом и $250 \times 10^{9} /$ л у пациентов без стеатогепатоза; $\mathrm{p}=0,0183)$. В целом ряде исследований также продемонстрирована связь между тромбоцитарной активностью и уровнем тромбоцитов со стеатогепатозом и другими состояниями, тесно связанными с инсулинорезистентностью, в том числе с метаболическим синдромом [22-25].

Ассоциации между наличием вируса Эпштейна - Барр, цитомегаловирусной инфекции, вируса простого герпеса в биоптатах толстой кишки и наличием стеатоза / стеатогепатита мы не обнаружили. Однако был показан высокий риск наличия вируса Эпштейна - Барр у пациентов 
Таблица 2. Сравнительная характеристика больных язвенным колитом в зависимости от наличия (1) или отсутствия (2) неалкогольного стеатоза / стеатогепатита $(\mathrm{n}=44)$

\begin{tabular}{llll}
\hline Показатель & $1(\mathrm{n}=15)$ & $2(\mathrm{n}=29)$ & $p$ \\
\hline Возраст, годы (медиана; $\mathrm{Q}_{1} / \mathrm{Q}_{3}$ ) & $61 ; 48 / 63$ & $29 ; 26 / 41,5$ & 0,0069 \\
Доля пациентов, получавших глюкокортикостероиды, $\%$ & 53 & 46 & 0,1186 \\
Доля пациентов, получавших биологическую терапию, $\%$ & 15 & 21 & 0,0132 \\
Индекс массы тела (медиана; $\mathrm{Q}_{1} / \mathrm{Q}_{3}$ ) & 28,$6 ; 22,2 / 32,2$ & 20,$4 ; 18,9 / 24,4$ & 0,0457 \\
Минимальный уровень гемоглобина, г/л (медиана; $\left.\mathrm{Q}_{1} / \mathrm{Q}_{3}\right)$ & $125 ; 112 / 137$ & $129 ; 115 / 138$ & 0,1174 \\
Максимальное значение СОЭ, мм/ч (медиана; $\left.\mathrm{Q}_{1} / \mathrm{Q}_{3}\right)$ & $19 ; 10 / 36$ & $10 ; 4 / 20$ & 0,1077 \\
Уровень холестерина, мкмоль/л (медиана; $\left.\mathrm{Q}_{1} / \mathrm{Q}_{3}\right)$ & 5,$37 ; 4,9 / 5,7$ & 4,$8 ; 4,4 / 5,3$ & 0,8495 \\
\hline
\end{tabular}

СОЭ - скорость оседания эритроцитов

Таблица 3. Сравнительная характеристика больных болезнью Крона в зависимости от наличия (1) или отсутствия (2) неалкогольного стеатоза / стеатогепатита $(n=49)$

\begin{tabular}{|c|c|c|c|}
\hline Показатель & $1(n=19)$ & $2(n=30)$ & $p$ \\
\hline Возраст, годы (медиана; $\mathrm{Q}_{1} / \mathrm{Q}_{3}$ ) & $51 ; 33 / 54$ & 31,$5 ; 25,5 / 37$ & 0,4835 \\
\hline Доля пациентов, получавших азатиоприн, \% & 23 & 38 & 0,0123 \\
\hline Доля пациентов, получавших биологическую терапию, \% & 25 & 10 & 0,0123 \\
\hline Максимальное снижение массы тела, кг (медиана; $\mathrm{Q}_{1} / \mathrm{Q}_{3}$ ) & $10 ; 0 / 15$ & $4 ; 0 / 10$ & 0,3178 \\
\hline Индекс массы тела (медиана; $\mathrm{Q}_{1} / \mathrm{Q}_{3}$ ) & 24,$1 ; 21 / 30,3$ & 20,$3 ; 18,6 / 23$ & 0,3663 \\
\hline Минимальный уровень гемоглобина, г/л (медиана; $\mathrm{Q}_{1} / \mathrm{Q}_{3}$ ) & $114 ; 98 / 125$ & $119 ; 109 / 136$ & 0,0895 \\
\hline Максимальная СОЭ, мм/ч (медиана; $\mathrm{Q}_{1} / \mathrm{Q}_{3}$ ) & $28 ; 10 / 35$ & $11 ; 4 / 22$ & 0,1039 \\
\hline Доля пациентов, перенесших оперативное лечение, \% & 25 & 24,14 & 0,4866 \\
\hline Уровень холестерина, мкмоль/л (медиана; $\mathrm{Q}_{1} / \mathrm{Q}_{3}$ ) & 4,$24 ; 3,7 / 4,9$ & 4,$67 ; 4,3 / 5,7$ & 0,4623 \\
\hline Уровень тромбоцитов в крови, × 109/л (медиана; $\mathrm{Q}_{1} / \mathrm{Q}_{3}$ ) & $287 ; 192 / 420$ & $250 ; 180 / 379$ & 0,0183 \\
\hline
\end{tabular}

СОЭ - скорость оседания эритроцитов

с ВЗК, получавших глюкокортикостероиды в анамнезе (ОШ 11,06; 95\% ДИ 2,62-46,83).

Анализ 7 наблюдательных исследований из баз данных Medline, Embase и Cochrane, охвативший 1610 больных В3К, не выявил серьезных связей между НАЖБП и принимаемыми медикаментами: биологическими агентами (ОШ 0,85; 95\% ДИ 0,49-1,46), иммуномодуляторами (ОШ 1,19; 95\% ДИ 0,7-2,01), метотрексатом (ОШ 3,62; 95\% ДИ 0,48-27,39) и стероидами (ОШ 1,24; 95\% ДИ 0,85$1,82)[26]$.

Вместе с тем в недавно опубликованном исследовании стеатоз печени рассматривается как фактор, ассоциированный с лекарственным поражением печени (ОШ 1,13; 95\% ДИ 1,02-1,26), наряду с более высоким ИМТ (ОШ 10,61; 95\% ДИ 2,22-50,7), большей длительностью течения ВЗК (ОШ 1,07; 95\% ДИ 1-1,15) и монотерапией инфликсимабом (ОШ 4,57; 95\% ДИ 1,33-15,7) [27].

A. Sartini и соавт. [20] в своем исследовании, включающем 223 больных НАЖБП (78 с ВЗК и 145 без патологии кишечника), отмечают определенные ассоциации. При наличии ВЗК реже встречается гиперферментемия, ниже ИМТ, меньше окружность талии, реже диагностирован метаболический синдром. Более тяжелое течение ВЗК чаще сопровождается стеатозом печени $(32,1$ против $16,6 \% ; \mathrm{p}=0,01)$. К независимым 
факторам риска наличия стеатоза относятся следующие: более одного обострения патологии кишечника в год (ОШ 17,3; 95\% ДИ 3,6-84), применение хирургического лечения (ОШ 15,1; 95\% ДИ 3,1-73,7), большая протяженность поражения (ОШ 19,4; 95\% ДИ 3,4-110,9). В этом исследовании установлен единственный протективный фактор в отношении стеатоза - прием препаратов, направленных против фактора некроза опухоли а (TNF- $\alpha$ ), особенно в отношении гиперферментемии (ОШ 0,15; 95\% ДИ 0-0,8; p=0,02).

В недавней работе D.E. Bosch и M.M. Yeh [28] протективным фактором стеатоза печени у больных ВЗК оказался первичный склерозирующий холангит, при этом как у больных без трансплантации, так и после трансплантации печени. Стеатоз печени при ВЗК ассоциирован с ИМТ, холестерином, артериальной гипертензией, сахарным диабетом, злоупотреблением алкоголем.

Наши собственные данные показали ассоциацию между стеатозом/стеатогепатитом у пациентов с ВЗК и наличием СИБР. Так, стеатоз у пациентов с ВЗК и СИБР встречался чаще в 39\% случаев, чем у пациентов с ВЗК без СИБР в 22\% (ОШ 2,34; 95\% ДИ 1,4-4,8; $\mathrm{p}=0,021)$. Это относилось к больным и БК, и ЯК. У пациентов с БК и СИБР стеатоз встречался в 38,9\%, тогда как в отсутствие СИБР - в 22,9\% (ОШ 2,1; 95\% ДИ 0,8-6; $\mathrm{p}=0,2)$. У пациентов с ЯК при наличии СИБР стеатоз регистрировали в $40 \%$, без такового - в 20,9\% (ОШ 2,5; 95\% ДИ 0,9-6,8; p=0,083).

Синдром цитолиза при наличии стеатоза (неалкогольный стеатогепатит) чаще встречался у пациентов с ВЗК и СИБР - в 31,5\%, в то время как без СИБР - в 16,5\% случаев (ОШ 2,3; 95\% ДИ $1-5 ; \mathrm{p}=0,091)$. Закономерности эти касались больных как с БК, так и с ЯК.

A.J. Wigg и соавт. [29] доказали в эксперименте, что системная эндотоксемия, вызванная СИБР, способствует продукции TNF- $\alpha$ и стеатогепатиту. Эндотоксин, образующийся в просвете тонкой кишки при СИБР, играет важную роль в патогенезе НАЖБП, влияя на клетки Купфера и провоцируя продукцию TNF- $\alpha$. Результаты исследования с участием 22 пациентов с НАЖБП показали, что уровень TNF- $\alpha$ вдвое выше, чем в контрольной группе. Частота встречаемости СИБР у пациентов с НАЖБП в этом исследовании составила 50\%. Авторы считают, что повышенная продукция TNF- $\alpha$ связана с эндотоксемией вследствие СИБР [29]. Позже A. Fialho и соавт. [30] на основании детального исследования 372 больных НАЖБП выявили высокую частоту СИБР - 37,9\%.
Проведенная нами оценка выраженности фиброза у больных со стеатозом показала, что у больных ВЗК со стеатозом индекс фиброза FIB4 сильно коррелировал с СОЭ ( $\mathrm{r}=0,765 ; \mathrm{p}=0,0343)$.

По данным С. Saroli Palumbo и соавт. [9], выраженность фиброза печени у больных ВЗК независимо ассоциирована с возрастом (ОШ 1,38; 95\% ДИ 1,12-1,64) и высоким ИМТ (ОШ 1,14; 95\% ДИ 1,07-1,23).

Таким образом, имеющиеся на сегодня данные указывают на дальнейшую необходимость тщательного изучения ассоциированных с этой патологией факторов. В литературе ожирение и связанные с ним состояния, такие как НАЖБП, рассматриваются как проявления системного воспалительного процесса. Так, по результатам проспективного наблюдательного исследования, проводившегося с 2005 по 2010 г., установлена взаимосвязь между наличием системного воспалительного процесса и рисками развития в будущем НАЖБП [31]. Мультивариантный регрессионный анализ с поправкой на возраст, проявления метаболического синдрома выявил взаимосвязь между наличием у пациента высокого исходно уровня лейкоцитов и развитием стеатогепатоза через 5 лет (ОШ 1,85; 95\% ДИ 1,1-3,1 для женщин и ОШ 1,68; 95\% ДИ 1,08-2,61 для мужчин; $\mathrm{p}<0,004)$. Похожие результаты получены в исследовании S. Wang и соавт., в котором изучалась взаимосвязь между уровнем лейкоцитов и риском развития стеатогепатоза [32].

Другим важным фактором в формировании стеатогепатоза у пациентов с ВЗК считается действие лекарственных препаратов, применяемых для терапии основного заболевания. Согласно результатам исследования, посвященного изучению факторов риска формирования НАЖБП у больных ВЗК, у пациентов, получающих глюкокортикостероиды, частота НАЖБП была выше, чем в группе больных ВЗК, не получающих глюкокортикостероиды (25 против 9,2\%; $\mathrm{p}=0,002)$ [33]. Среди пациентов без НАЖБП частота применения анти-TNF-a была выше по сравнению с группой с НАЖБП (29,7 против $17,1 \% ; \mathrm{p}=0,048)$. Это предположительно связано с возможным протективным свойством анти-TNF- $\alpha$ в отношении развития стеатогепатоза [34]. Весьма вероятно, что изменения микробиоты при ВЗК вносят свой вклад в развитие стеатогепатоза [35-37].

\section{Заключение}

Полученные нами результаты согласуются с данными других исследований, посвященных 


\section{Конфликт интересов}

Авторы декларируют отсутствие явных и потенциальных конфликтов интересов, связанных с публикацией настоящей статьи.

\section{Финансирование}

Работа проведена без привлечения дополнительного финансирования со стороны третьих лиц. особенностям неалкогольного стеатогепатоза у больных ВЗК и установивших, что для этой категории пациентов характерны общепопуляционные факторы риска развития жирового гепатоза - возраст, ожирение, нарушение углеводного и липидного обменов. Вместе с тем у пациентов с ВЗК присутствуют особые факторы, ассоциированные с развитием неалкогольного стеатогепатоза и отражающие течение системного воспалительного процесса. В большинстве опубликованных работ пациенты с ВЗК рассматриваются как единая группа. Мы же показали, что факторы, ассоциированные со стеатогепатозом, могут различаться у больных ЯК и БК. Так, при ЯК наличие неалкогольного стеатоза ассоциировано с возрастом, а при БК - с уровнем тромбоцитов и получением биологической терапии. Это может указывать на определенные различия патогенеза стеатогепатоза у этих пациентов. Кроме того, мы выявили ассоциацию фиброза печени с уровнем СОЭ. Следует отметить, что при оценке уровня фиброза в большинстве работ (в том числе и в нашей) использовались неинвазивные индексы, отражающие фиброз печени лишь косвенно. В связи с этим было бы весьма интересно провести исследование с применением более точных диагностических методик.

Данные литературы позволяют также предположить существенный вклад кишечной микрофлоры в развитие стеатогепатоза у пациентов с ВЗК. Полученные нами результаты подтверждают эту гипотезу - в нашей работе СИБР был фактором, ассоциированным с развитием неалкогольного стеатогепатита. Публикаций, посвященных влиянию вируса Эпштейна - Барр, нам найти не удалось. В нашем исследовании не было выявлено значимой ассоциации между неалкогольным стеатогепатозом и наличием вируса Эпштейна - Барр. По всей видимости, этот вирус не имеет существенного самостоятельного значения в патогенезе неалкогольного стеатогепатоза. ()

\section{Литература}

1. Fiorucci S, Biagioli M, Distrutti E. Future trends in the treatment of non-alcoholic steatohepatitis. Pharmacol Res. 2018;134:289-98. doi: 10.1016/j.phrs.2018.07.014.

2. Karim MF, Al-Mahtab M, Rahman S, Debnath CR. Non-alcoholic Fatty Liver Disease (NAFLD) - a review. Mymensingh Med J. 2015;24(4):873-80.

3. Звенигородская ЛА, Чурикова АА. Особенности алкогольной и неалкогольной болезни печени. Экспериментальная и клиническая гастроэнтерология. 2012;(11):34-7.

4.Подымова СД. Эволюция представлений о неалкогольной жировой болезни печени. Экспериментальная и клиническая гастроэнтерология. 2009;(4):4-12.

5. Соловьева АВ, Гуль ИС. К вопросу о гепатобилиарной патологии при метаболическом синдроме. Медицинский альманах. 2015;(1): 66-9.

6. Chalasani N, Younossi Z, Lavine JE, Charlton M, Cusi K, Rinella M, Harrison SA, Brunt EM, Sanyal AJ. The diagnosis and management of nonalcoholic fatty liver disease: Practice guidance from the American Association for the Study of Liver Diseases. Hepatology. 2018;67(1):328-57. doi: 10.1002/hep.29367.

7. Быкова ГА, Хлынова ОВ, Туев АВ. Неалкогольная жировая болезнь печени и хроническая обструктивная болезнь легких: есть ли база для коморбидности? (обзор литературы). Пермский медицинский журнал. 2015;32(2):127-34.
8.Лю КМ. Коморбидное течение гастроэзофагеальной рефлюксной болезни и неалкогольной жировой болезни печени как фактор риска возникновения кардиальной патологии. Пермский медицинский журнал. 2017;34(1):100-6.

9. Saroli Palumbo $C$, Restellini $S$, Chao CY, Aruljothy A, Lemieux C, Wild G, Afif W, Lakatos PL, Bitton A, Cocciolillo S, Ghali P, Bessissow T, Sebastiani G. Screening for nonalcoholic fatty liver disease in inflammatory bowel diseases: a cohort study using transient elastography. Inflamm Bowel Dis. 2018 Jun 7. [Epub ahead of print]. doi: 10.1093/ibd/izy200.

10.Glassner K, Malaty HM, Abraham BP. Epidemiology and risk factors of nonalcoholic fatty liver disease among patients with inflammatory bowel disease. Inflamm Bowel Dis. 2017;23(6):998-1003. doi: 10.1097/ MIB.00000000000001085.

11. Tornai T, Palyu E, Vitalis Z, Tornai I, Tornai $D$, Antal-Szalmas P, Norman GL, Shums Z, Veres G, Dezsofi A, Par G, Par A, Orosz P, Szalay F, Lakatos PL, Papp M. Gut barrier failure biomarkers are associated with poor disease outcome in patients with primary sclerosing cholangitis. World J Gastroenterol. 2017;23(29):5412-21. doi: 10.3748/wjg.v23.i29.5412.

12. Bischoff SC, Barbara G, Buurman W, Ockhuizen T, Schulzke JD, Serino M, Tilg H, Watson A, Wells JM. Intestinal permeability - a new target for disease prevention and therapy. BMC Gastroenterol. 2014;14:189. doi: 10.1186/ s12876-014-0189-7.
13. Michielan A, D'Incà R. Intestinal permeability in inflammatory bowel disease: pathogenesis, clinical evaluation, and therapy of leaky gut. Mediators Inflamm. 2015;2015:628157. doi: 10.1155/2015/628157.

14. Ивашкин ВТ, Шелыгин ЮА, Халиф ИЛ, Белоусова ЕА, Шифрин ОС, Абдулганиева ДИ, Абдулхаков РА, Алексеева ОП, Алексеенко СА, Ачкасов СИ, Барановский АЮ, Болихов КВ, Валуйских ЕЮ, Варданян АВ, Веселов АВ, Веселов ВВ, Головенко АО, Головенко ОВ, Григорьев ЕГ, Губонина ИВ, Жигалова ТН, Кашников ВН, Кизова ЕА, Князев ОВ, Костенко НВ, Куляпин АВ, Морозова НА, Муравьев АВ, Низов АА, Никитина НВ, Николаева НH, Никулина НВ, Одинцова $\mathrm{AX}$, Осипенко МФ, Павленко ВВ, Парфенов АИ, Полуэктова ЕА, Потапов АС, Румянцев ВГ, Светлова ИО, Ситкин СИ, Тимербулатов ВМ, Ткачев АВ, Ткаченко ЕИ, Фролов СА, Хубезов ДА, Чашкова ЕЮ, Шапина МВ, Щукина ОБ, Яковлев АА. Клинические рекомендации Российской гастроэнтерологической ассоциации и Ассоциации колопроктологов России по диагностике и лечению язвенного колита. Колопроктология. 2017;(1):6-30.

15. Ивашкин ВТ, Шелыгин ЮА, Халиф ИЛ, Белоусова ЕА, Шифрин ОС, Абдулганиева ДИ, Абдулхаков РА, Алексеева ОП, Алексеенко СА, Ачкасов СИ, Барановский АЮ, Болихов КВ, Валуйских ЕЮ, Варданян АВ, Веселов $А B$, Веселов ВВ, Головенко АО, Головенко ОВ, Григорьев ЕГ, Губонина ИВ, Жигалова ТН, 
Кашников ВН, Кизова ЕА, Князев ОВ, Костенко НВ, Куляпин АВ, Морозова НA, Муравьев АВ, Низов АА, Никитина НВ, Николаева НН, Никулина НВ, Одинцова АХ, Осипенко МФ, Павленко ВВ, Парфенов АИ, Полуэктова ЕА, Потапов АС, Румянцев ВГ, Светлова ИО, Ситкин СИ, Тимербулатов ВМ, Ткачев АВ, Ткаченко ЕИ, Фролов СА, Хубезов ДА, Чашкова ЕЮ, Шапина МВ, Щукина ОБ, Яковлев АА. Клинические рекомендации российской гастроэнтерологической ассоциации и ассоциации колопроктологов России по диагностике и лечению болезни Крона. Колопроктология. 2017;(2):7-29.

16. Ивашкин ВТ, Маевская МВ, Павлов ЧС, Тихонов ИН, Широкова ЕН, Буеверов АО, Драпкина ОМ, Шульпекова ЮО, Цуканов ВВ, Маммаев СН, Маев ИВ, Пальгова ЛК. Клинические рекомендации по диагностике и лечению неалкогольной жировой болезни печени Российского общества по изучению печени и Российской гастроэнтерологической ассоциации. Российский журнал гастроэнтерологии, гепатологии, колопроктологии. 2016;(2):24-42.

17. Шептулина АФ, Широкова ЕН, Ивашкин ВТ. Неинвазивная диагностика фиброза печени: роль сывороточных маркеров. Российский журнал гастроэнтерологии, гепатологии, колопроктологии. 2015;(2):28-40.

18. Bargiggia S, Maconi G, Elli M, Molteni P, Ardizzone S, Parente F, Todaro I, Greco S, Manzionna G, Bianchi Porro G. Sonographic prevalence of liver steatosis and biliary tract stones in patients with inflammatory bowel disease: study of 511 subjects at a single center. J Clin Gastroenterol. 2003;36(5):417-20.

19. Principi M, lannone A, Losurdo G, Mangia M, Shahini E, Albano F, Rizzi SF, La Fortezza RF, Lovero R, Contaldo A, Barone M, Leandro G, lerardi $\mathrm{E}$, Di Leo A. Nonalcoholic fatty liver disease in inflammatory bowel disease: prevalence and risk factors. Inflamm Bowel Dis. 2018;24(7):1589-96. doi: 10.1093/ibd/izy051.

20. Sartini A, Gitto S, Bianchini M, Verga MC, Di Girolamo M, Bertani A, Del Buono M, Schepis F, Lei B, De Maria N, Villa E. Non-alcoholic fatty liver disease phenotypes in patients with inflammatory bowel disease. Cell Death Dis. 2018:9(2):87. doi: 10.1038/s41419-017-0124-2.

\section{References}

1. Fiorucci S, Biagioli M, Distrutti E. Future trends in the treatment of non-alcoholic steatohepatitis. Pharmacol Res. 2018;134:289-98. doi: 10.1016/j.phrs.2018.07.014

2. Karim MF, Al-Mahtab M, Rahman S, Debnath CR. Non-alcoholic Fatty Liver Disease (NAFLD) - a review. Mymensingh Med J. 2015;24(4):873-80.
21. Jamali R, Biglari M, Seyyed Hosseini SV, Shakouri Rad A, Kosari F. The Correlation Between Liver Fat Content and Ulcerative Colitis Disease Severity. Acta Med Iran. 2017;55(5):333-9.

22. Gasparyan AY, Ayvazyan L, Mikhailidis DP, Kitas GD. Mean platelet volume: a link between thrombosis and inflammation? Curr Pharm Des. 2011;17(1):47-58. doi: 10.2174/138161211795049804.

23. Celikbilek M, Gürsoy S, Deniz K, Karaman A Zararsiz G, Yurci A. Mean platelet volume in biopsy-proven non-alcoholic fatty liver disease. Platelets. 2013;24(3):194-9. doi: 10.3109/09537104.2012.688898.

24. Ahmadzadeh J, Mansorian B, Attari MM, Mohebbi I, Naz-Avar R, Moghadam K, GharehBagh SAK. The association between hematological parameters and metabolic syndrome in Iranian men: A single center large-scale study. Diabetes Metab Syndr. 2018;12(1):17-21. doi: 10.1016/j.dsx.2017.07.044.

25. Lohsoonthorn V, Jiamjarasrungsi W, Williams MA. Association of hematological parameters with clustered components of metabolic syndrome among professional and office workers in Bangkok, Thailand. Diabetes Metab Syndr. 2007;1(3):143-9. doi: 10.1016/j. dsx.2007.05.002.

26. Lapumnuaypol K, Kanjanahattakij N, Pisarcik D, Thongprayoon C, Wijarnpreecha K Cheungpasitporn W. Effects of inflammatory bowel disease treatment on the risk of nonalcoholic fatty liver disease: a meta-analysis. Eur J Gastroenterol Hepatol. 2018;30(8):854-60. doi: 10.1097/MEG.0000000000001144.

27. Koller T, Galambosova M, Filakovska S, Kubincova M, Hlavaty $T$, Toth J, Krajcovicova A, Payer J. Drug-induced liver injury in inflammatory bowel disease: 1-year prospective observational study. World J Gastroenterol. 2017;23(22):4102-11. doi: 10.3748/wjg.v23. i22.4102.

28. Bosch DE, Yeh MM. Primary sclerosing cholangitis is protective against nonalcoholic fatty liver disease in inflammatory bowel disease. Hum Pathol. 2017;69:55-62. doi: 10.1016/j. humpath.2017.09.008.

29. Wigg AJ, Roberts-Thomson IC, Dymock RB, McCarthy PJ, Grose RH, Cummins AG. The role of small intestinal bacterial overgrowth, intestinal permeability, endotoxaemia, and tumour necrosis factor alpha in the pathogenesis of non-alcoholic steatohepatitis. Gut. 2001;48(2): 206-11. doi: 10.1136/gut.48.2.206.

30. Fialho A, Fialho A, Thota P, McCullough AJ, Shen B. Small intestinal bacterial overgrowth is associated with non-alcoholic fatty liver disease. J Gastrointestin Liver Dis. 2016;25(2): 159-65. doi: 10.15403/jgld.2014.1121.252.iwg.

31. Chung GE, Yim JY, Kim D, Kwak MS, Yang Jl, ChungSJ,Yang SY, Kim JS. Associations between white blood cell count and the development of incidental nonalcoholic fatty liver disease. Gastroenterol Res Pract. 2016;2016:7653689. doi: $10.1155 / 2016 / 7653689$.

32. Wang S, Zhang C, Zhang G, Yuan Z, Liu Y, Ding L, Sun $X$, Jia $H$, Xue F. Association between white blood cell count and non-alcoholic fatty liver disease in urban Han Chinese: a prospective cohort study. BMJ Open. 2016;6(6):e010342. doi: 10.1136/bmjopen-2015-010342.

33. Sourianarayanane A, Garg G, Smith TH, Butt MI, McCullough AJ, Shen B. Risk factors of non-alcoholic fatty liver disease in patients with inflammatory bowel disease. J Crohns Colitis. 2013;7(8):e279-85. doi: 10.1016/j. crohns.2012.10.015.

34. Barbuio R, Milanski M, Bertolo MB, Saad MJ, Velloso LA. Infliximab reverses steatosis and improves insulin signal transduction in liver of rats fed a high-fat diet. J Endocrinol. 2007;194(3):539-50. doi: 10.1677/JOE-070234 .

35. Malaguarnera $M$, Vacante $M$, Antic $T$, Giordano $M$, Chisari G, Acquaviva R, Mastrojeni S, Malaguarnera G, Mistretta A, Li Volti G, Galvano F. Bifidobacterium longum with fructo-oligosaccharides in patients with non alcoholic steatohepatitis. Dig Dis Sci. 2012;57(2):545-53. doi: 10.1007/s10620-011-1887-4.

36. Daubioul CA, Horsmans $Y$, Lambert $P$, Danse $E_{t}$ Delzenne NM. Effects of oligofructose on glucose and lipid metabolism in patients with nonalcoholic steatohepatitis: results of a pilot study. Eur J Clin Nutr. 2005;59(5):723-6. doi: 10.1038/sj.ejcn.1602127.

37. Mofidi F, Poustchi $H$, Yari $Z$, Nourinayyer B, Merat S, Sharafkhah M, Malekzadeh R, Hekmatdoost A. Synbiotic supplementation in lean patients with non-alcoholic fatty liver disease: a pilot, randomised, double-blind, placebo-controlled, clinical trial. Br J Nutr. 2017;117(5):662-8. doi: 10.1017/ S0007114517000204.

3. Zvenigorodskaya LA, Churikova AA. Particulars of alcoholic and non-alcoholic liver disease. Experimental and Clinical Gastroenterology Journal. 2012;(11):34-7. Russian.

4. Podymova SD. Evolution of the understanding of non-alcoholic fatty liver disease. Expermental and Clinical Gastroenterology Journal. 2009:(4):4-12. Russian.
5. Solovieva AV, Gul IS. On the question of hepatobiliary pathology in the case of metabolic syndrome. Medical Almanac. 2015;(1):66-9. Russian.

6. Chalasani N, Younossi Z, Lavine JE, Charlton M, Cusi K, Rinella M, Harrison SA, Brunt EM, Sanyal AJ. The diagnosis and management of nonalcoholic fatty liver disease: Practice guidance 
from the American Association for the Study of Liver Diseases. Hepatology. 2018;67(1):328-57. doi: 10.1002/hep.29367.

7. Bykova GA, Khlynova OV, Tuev AV. Nonalcoholic fatty hepatic disease and chronic obstructive pulmonary disease: is there a basis for comorbidity? (review of literature). Perm Medical Journal. 2015;32(2):127-34. Russian.

8. Lyu KM. Comorbid course of gastroesophageal reflux disease and nonalcoholic amyloid liver disease as risk factor for cardiac pathology. Perm Medical Journal. 2017;34(1):100-6. Russian.

9. Saroli Palumbo C, Restellini S, Chao CY, Aruljothy A, Lemieux C, Wild G, Afif W, Lakatos PL, Bitton A, Cocciolillo S, Ghali P, Bessissow T, Sebastiani G. Screening for nonalcoholic fatty liver disease in inflammatory bowel diseases: a cohort study using transient elastography. Inflamm Bowel Dis. 2018 Jun 7. [Epub ahead of print]. doi: 10.1093/ibd/izy200.

10. Glassner K, Malaty HM, Abraham BP. Epidemiology and risk factors of nonalcoholic fatty liver disease among patients with inflammatory bowel disease. Inflamm Bowel Dis. 2017;23(6):998-1003. doi: 10.1097/ MIB. 0000000000001085 .

11. Tornai T, Palyu E, Vitalis Z, Tornai I, Tornai D, Antal-Szalmas $P$, Norman GL, Shums Z, Veres G, Dezsofi A, Par G, Par A, Orosz P, Szalay F, Lakatos $\mathrm{PL}$, Papp M. Gut barrier failure biomarkers are associated with poor disease outcome in patients with primary sclerosing cholangitis. World J Gastroenterol. 2017;23(29):5412-21. doi: 10.3748/wjg.v23.i29.5412.

12. Bischoff SC, Barbara G, Buurman W, Ockhuizen T, Schulzke JD, Serino M, Tilg H, Watson A, Wells JM. Intestinal permeability - a new target for disease prevention and therapy. BMC Gastroenterol. 2014;14:189. doi: 10.1186/ s12876-014-0189-7.

13. Michielan A, D'Incà R. Intestinal permeability in inflammatory bowel disease: pathogenesis, clinical evaluation, and therapy of leaky gut. Mediators Inflamm. 2015;2015:628157. doi: $10.1155 / 2015 / 628157$.

14. Ivashkin VT, Shelygin YuA, Khalif IL, Belousova EA, Shifrin OS, Abdulganieva DI, Abdulkhakov RA, Alekseeva OP, Alekseenko SA, Achkasov SI, Baranovskiy AYu, Bolikhov KV, Valuyskikh EYu, Vardanyan AV, Veselov AV, Veselov VV, Golovenko AO, Golovenko OV, Grigor'ev EG, Gubonina IV, Zhigalova TN, Kashnikov VN, Kizova EA, Knyazev OV, Kostenko NV, Kulyapin AV, Morozova NA, Murav'ev AV, Nizov AA, Nikitina NV, Nikolaeva NN, Nikulina NV, Odintsova AKh, Osipenko MF, Pavlenko VV, Parfenov Al, Poluektova EA, Potapov AS, Rumyantsev VG, Svetlova IO, Sitkin SI, Timerbulatov VM, Tkachev AV, Tkachenko El, Frolov SA, Khubezov DA, Chashkova EYu, Shapina MV, Shchukina OB, Yakovlev AA. Clinical guide of Russian Association of Gastroenterology and Russian Association of Coloproctology on di- agnostics and treatment of ulcerative colitis. Koloproktologia. 2017;(1):6-30. Russian.

15. Ivashkin VT, Shelygin YuA, Khalif IL, Belousova EA, Shifrin OS, Abdulganieva DI, Abdulkhakov RA, Alekseeva OP, Alekseenko SA, Achkasov SI, Baranovskiy AYu, Bolikhov KV, Valuyskikh EYu, Vardanyan AV, Veselov AV, Veselov VV, Golovenko AO, Golovenko OV, Grigor'ev EG, Gubonina IV, Zhigalova TN, Kashnikov VN, Kizova EA, Knyazev OV, Kostenko NV, Kulyapin AV, Morozova NA, Murav'ev AV, Nizov AA, Nikitina NV, Nikolaeva NN, Nikulina NV, Odintsova AKh, Osipenko MF, Pavlenko VV, Parfenov Al, Poluektova EA, Potapov AS, Rumyantsev VG, Svetlova IO, Sitkin SI, Timerbulatov VM, Tkachev AV, Tkachenko El, Frolov SA, Khubezov DA, Chashkova EYu, Shapina MV, Shchukina OB, Yakovlev AA. Clinical guide of Russian Association of Gastroenterology and Russian Association of Coloproctology on diagnostics and treatment of Crohn's disease. Koloproktologia. 2017;(2):7-29. Russian.

16. Ivashkin VT, Mayevskaya MV, Pavlov ChS, Tikhonov IN, Shirokova YeN, Buyeverov AO, Drapkina OM, Shulpekova YuO, Tsukanov VV, Mammayev SN, Mayev IV, Palgova LK. Diagnostics and treatment of non-alcoholic fatty liver disease: clinical guidelines of the Russian Scientific Liver Society and the Russian gastroenterological association. Russian Journal of Gastroenterology, Hepatology, Coloproctology. 2016;(2):24-42. Russian.

17. Sheptulina AF, Shirokova YeN, Ivashkin VT. Non-invasive of liver fibrosis diagnostics: the role of serum markers. Russian Journal of Gastroenterology, Hepatology, Coloproctology. 2015;(2):28-40. Russian.

18. Bargiggia S, Maconi G, Elli M, Molteni P, Ardizzone S, Parente F, Todaro I, Greco S, Manzionna G, Bianchi Porro G. Sonographic prevalence of liver steatosis and biliary tract stones in patients with inflammatory bowel disease: study of 511 subjects at a single center. J Clin Gastroenterol. 2003;36(5):417-20.

19. Principi $M$, lannone $A$, Losurdo $G$, Mangia $M$, Shahini E, Albano F, Rizzi SF, La Fortezza RF, Lovero R, Contaldo A, Barone M, Leandro G, lerardi $\mathrm{E}$, Di Leo A. Nonalcoholic fatty liver disease in inflammatory bowel disease: prevalence and risk factors. Inflamm Bowel Dis. 2018;24(7):1589-96. doi: 10.1093/ibd/izy051.

20. Sartini A, Gitto S, Bianchini M, Verga MC, Di Girolamo M, Bertani A, Del Buono M, Schepis F, Lei B, De Maria N, Villa E. Non-alcoholic fatty liver disease phenotypes in patients with inflammatory bowel disease. Cell Death Dis. 2018;9(2):87. doi: 10.1038/s41419-017-0124-2.

21. Jamali R, Biglari M, Seyyed Hosseini SV, Shakouri Rad A, Kosari F. The Correlation Between Liver Fat Content and Ulcerative Colitis Disease Severity. Acta Med Iran. 2017;55(5):333-9.

22. Gasparyan AY, Ayvazyan L, Mikhailidis DP, Kitas GD. Mean platelet volume: a link between thrombosis and inflammation? Curr Pharm Des. 2011;17(1):47-58. doi: 10.2174/138161211795049804.

23. Celikbilek M, Gürsoy S, Deniz K, Karaman A, Zararsiz G, Yurci A. Mean platelet volume in biopsy-proven non-alcoholic fatty liver disease. Platelets. 2013;24(3):194-9. doi: 10.3109/09537104.2012.688898.

24. Ahmadzadeh J, Mansorian B, Attari MM, Mohebbi I, Naz-Avar R, Moghadam K, GharehBagh SAK. The association between hematological parameters and metabolic syndrome in Iranian men: A single center large-scale study. Diabetes Metab Syndr. 2018;12(1):17-21. doi: 10.1016/j.dsx.2017.07.044.

25. Lohsoonthorn V, Jiamjarasrungsi W, Williams MA. Association of hematological parameters with clustered components of metabolic syndrome among professional and office workers in Bangkok, Thailand. Diabetes Metab Syndr. 2007;1(3):143-9. doi: 10.1016/j. dsx.2007.05.002.

26. Lapumnuaypol K, Kanjanahattakij N, Pisarcik D, Thongprayoon C, Wijarnpreecha K, Cheungpasitporn W. Effects of inflammatory bowel disease treatment on the risk of nonalcoholic fatty liver disease: a meta-analysis. Eur J Gastroenterol Hepatol. 2018;30(8):854-60. doi: 10.1097/MEG.0000000000001144.

27. Koller T, Galambosova M, Filakovska S, Kubincova M, Hlavaty T, Toth J, Krajcovicova A, Payer J. Drug-induced liver injury in inflammatory bowel disease: 1-year prospective observational study. World J Gastroenterol. 2017;23(22):4102-11. doi: 10.3748/wjg.v23. i22.4102.

28. Bosch DE, Yeh MM. Primary sclerosing cholangitis is protective against nonalcoholic fatty liver disease in inflammatory bowel disease. Hum Pathol. 2017;69:55-62. doi: 10.1016/j. humpath.2017.09.008.

29. Wigg AJ, Roberts-Thomson IC, Dymock RB, McCarthy PJ, Grose RH, Cummins AG. The role of small intestinal bacterial overgrowth, intestinal permeability, endotoxaemia, and tumour necrosis factor alpha in the pathogenesis of non-alcoholic steatohepatitis. Gut. 2001;48(2): 206-11. doi: 10.1136/gut.48.2.206.

30. Fialho A, Fialho A, Thota P, McCullough AJ, Shen B. Small intestinal bacterial overgrowth is associated with non-alcoholic fatty liver disease. J Gastrointestin Liver Dis. 2016;25(2): 159-65. doi: 10.15403/jgld.2014.1121.252.iwg.

31. Chung GE, Yim JY, Kim D, Kwak MS, Yang Jl, Chung SJ, Yang SY, Kim JS. Associations between white blood cell count and the development of incidental nonalcoholic fatty liver disease. Gastroenterol Res Pract. 2016;2016:7653689. doi: 10.1155/2016/7653689.

32. Wang S, Zhang C, Zhang G, Yuan Z, Liu Y, Ding L, Sun $X$, Jia H, Xue F. Association between white blood cell count and non-alcoholic fatty liver disease in urban Han Chinese: a prospective 
cohort study. BMJ Open. 2016;6(6):e010342. doi: 10.1136/bmjopen-2015-010342.

33. Sourianarayanane A, Garg G, Smith $T H$, Butt MI, McCullough AJ, Shen B. Risk factors of non-alcoholic fatty liver disease in patients with inflammatory bowel disease. J Crohns Colitis. 2013;7(8):e279-85. doi: 10.1016/j. crohns.2012.10.015.

34. Barbuio R, Milanski M, Bertolo MB, Saad MJ, Velloso LA. Infliximab reverses steatosis and improves insulin signal transduction in liver of rats fed a high-fat diet. J Endocrinol.
2007;194(3):539-50. doi: 10.1677/JOE-070234.

35. Malaguarnera $M$, Vacante $M$, Antic $T$, Giordano $M$, Chisari $G$, Acquaviva R, Mastrojeni $S$, Malaguarnera G, Mistretta A, Li Volti G, Galvano F. Bifidobacterium longum with fructo-oligosaccharides in patients with non alcoholic steatohepatitis. Dig Dis Sci. 2012;57(2):545-53. doi: 10.1007/s10620-011-1887-4.

36. Daubioul CA, Horsmans Y, Lambert P, Danse E, Delzenne NM. Effects of oligofructose on glu- cose and lipid metabolism in patients with nonalcoholic steatohepatitis: results of a pilot study. Eur J Clin Nutr. 2005;59(5):723-6. doi: 10.1038/sj.ejcn.1602127.

37. Mofidi F, Poustchi H, Yari Z, Nourinayyer B, Merat S, Sharafkhah M, Malekzadeh R, Hekmatdoost A. Synbiotic supplementation in lean patients with non-alcoholic fatty liver disease: a pilot, randomised, double-blind, placebo-controlled, clinical trial. Br J Nutr. 2017;117(5): 662-8. doi: 10.1017/S0007114517000204.

\title{
Prevalence and characteristics of non-alcoholic steatohepatitis in patients with inflammatory bowel disease in the Novosibirsk region: a cross-sectional, one center study in 245 patients
}

\author{
Ya.A. Krasner ${ }^{1}$ Yu.A. Kulygina' • E.Yu. Valuyskikh² ${ }^{2}$ \\ M.F. Osipenko'
}

Rationale: Recently, the incidence of inflammatory bowel disease (IBD) and non-alcoholic steatohepatosis has increased in developed countries. Also, there are fundamental prerequisites to mutually negative influence of these diseases. Therefore, evaluation of the characteristics of non-alcoholic steatohepatosis in patients with IBD is of practical interest. Aim: To identify particulars of non-alcoholic steatohepatosis/steatohepatitis in IBD patients. Materials and methods: This cross-sectional study included 245 patients aged from 18 to 77 years from the IBD Registry the Novosibirsk State Medical University and the State Scientific-Research Institute of Physiology and Basic Medicine (Novosibirsk, Russian Federation). Within one year before the study entry, the patients were assessed by abdominal ultrasound and diagnosed with steatohepatosis. All patients were examined clinically with measurement of their antropometric parameters and underwent laboratory assessments, including hematology tests, alanine and aspartate transaminases, creatinine, triglycerides and total cholesterol measurements. Patients with viral and autoimmune hepatitis, alcoholic liver disease, primary sclerosing cholangitis, iron and copper metabolism disorders were excluded. In the colon biopsy samples of 45 patients, Epstein-Barr virus, cytomegalovirus, herpes simplex virus were identified by polymerase chain reaction. One hundred and fifty two (152) patients were also assessed for small intestine bacterial overgrowth (SIBO) syndrome. Results: The prevalence of steatosis in patients with Crohn's disease (CD) was $34.2 \%$, and in those with ulcerative colitis (UC), 30.4\%. IBD patients with steatohepatosis were more likely to have received steroids (63.6\% vs. $53 \%, p=0.0006)$, had greater IBD duration (median 5.9 years, $\mathrm{Q}_{1} / \mathrm{Q}_{3}, 2.7 / 12.9$ years vs. $4.5,2.9 / 8.5$ years; $p=0.0324$ ) and a higher body mass index (median $24.1, Q_{1} / Q_{3}, 21.4 / 29.9$ vs. 21 , 18.6/23.5; $p=0.0336$ ). UC patients with non-alcoholic steatosis/steatohepatitis were older (odds ratio [OR] for the age above 40 years, 1.46; 95\% confidence interval $[\mathrm{CI}] 1.01-2.1)$. CD patients with steatohepatosis had higher platelet counts (me$\operatorname{dian} 287 \times 10^{9} / \mathrm{L}, \mathrm{Q}_{1} / \mathrm{Q}_{3}, 192 \times 10^{9} / \mathrm{L} / 420 \times 10^{9} / \mathrm{L}$ vs. $\left.250 \times 10^{\circ} / \mathrm{L}, \quad 180 \times 10^{9} / \mathrm{L} / 379 \times 10^{9} / \mathrm{L} ; \quad \mathrm{p}=0.0183\right)$. SIBO was a risk factor for the development of steatohepatosis in IBD patients (OR 2.34, 95\% C 1.4-4.8, $p=0.021)$. Conclusion: The study has identified the differences in the steatohepatosis-associated factors in UC and CD patients. There is a link between the presence of SIBO and the risk of steatohepatosis development in IBD patients.

Key words: inflammatory bowel disease, Crohn's disease, ulcerative colitis, steatohepatosis, microflora

For citation: Krasner YaA, Kulygina YuA, Valuyskikh EYu, Osipenko MF. Prevalence and characteristics of non-alcoholic steatohepatitis in patients with inflammatory bowel disease in the Novosibirsk region: a cross-sectional, one center study in 245 patients. Almanac of Clinical Medicine. 2018;46(5):464-73. doi: 10.18786/2072-0505-2018-46-5-464-473.

Received 1 August 2018; accepted 2 October 2018
Yakov A. Krasner - Assistant, Chair of Propaedeutics of Internal Diseases

$\triangle$ 5/1-13 Kotovskogo ul., Novosibirsk, 630078 Russian Federation. Tel.: +7 (913) 9077766 E-mail: yakov.krasner@yandex.ru

Yulia A. Kulygina - Assistant, Chair of Propaedeutics of Internal Diseases

Ekaterina Yu. Valuyskikh - MD, PhD, Head of Department of Therapy ${ }^{2}$

Marina F. Osipenko - MD, PhD, Professor, Head of the Chair of Propaedeutics of Internal Diseases'

\section{Conflict of interests}

The authors declare no conflicts of interests.
${ }^{1}$ Novosibirsk State Medical University; 52 Krasnyy prospekt, Novosibirsk, 630091, Russian Federation

${ }^{2}$ State Scientific-Research Institute of Physiology and Basic Medicine; 4 Timakova ul., Novosibirsk, 630117, Russian Federation 